\title{
The Impact of Fentanyl Matrix on Pain and Function in Spinal Disorder-Related Chronic Pain: An Open Label Trial in Korea
}

\author{
Jae Hyup Lee ${ }^{1}$, Sung-Cheol Yun², Jung-Gil Lee ${ }^{3}$, Kyoung Hyo Choi ${ }^{4}$, \\ Dong-Soo Kim ${ }^{5}$, Kook-Jin Chung ${ }^{6}$, Ye-Soo Park , Whan Eoh ${ }^{8}$ \\ 'Department of Orthopedic Surgery, Institute of Medical and Biological Engineering, Seoul National University Medical \\ Research Center, SMG-SNU Boramae Medical Center, Seoul National University College of Medicine, Seoul, Korea \\ ${ }^{2}$ Department of Preventive Medicine, Asan Medical Center, University of Ulsan College of Medicine, Seoul, Korea \\ ${ }^{3}$ Department of Neurosurgery, Chonnam National University Medical School, Gwangju, Korea \\ ${ }^{4}$ Department of Rehabilitation Medicine, Asan Medical Center, University of Ulsan College of Medicine, Seoul, Korea \\ ${ }^{5}$ Department of Orthopedic Surgery, Chungbuk National University Hospital, \\ Chungbuk National University College of Medicine, Cheongju, Korea \\ ${ }^{6}$ Department of Orthopedic Surgery, Kangnam Sacred Heart Hospital, Hallym University College of Medicine, Seoul, Korea \\ 'Department of Orthopedic Surgery, Hanyang University Guri Hospital, \\ Hanyang University College of Medicine, Guri, Korea \\ ${ }^{8}$ Department of Neurosurgery, Samsung Medical Center, Sungkyunkwan University School of Medicine, Seoul, Korea
}

Study Design: This is a multicenter, open-label prospective, non interventional study.

Purpose: We wanted to evaluate the impact of fentanyl matrix on the pain and function of patients with spinal disorderrelated chronic, non-malignant pain.

Overview of Literature: Patients with severe non-malignant chronic low back pain may require opioid analgesics for effective pain management.

Methods: A total of 1,576 patients with severe pain (numeric rating scale $=7$ ) were evaluated for their pain intensity at the initial visit and at weeks 4 and 8 (Visits 1, 2, and 3, respectively). Disturbances in sleep, daily living and social activities, the Oswestry Disability Index (ODI), the researchers' and patients' global assessment and the patients' treatment preference were also assessed.

Results: The pain intensity score significantly decreased from 8.1 at Visit 1 to 5.4 and 4.4 at Visits 2 and 3, respectively. Sleep disturbance also significantly decreased and the extent of disturbance of daily and social activities was also significantly improved. The ODI significantly decreased from $61.9 \%$ to $45.8 \%$ and $38.2 \%$ at Visits 1, 2, and 3, respectively. Adverse events were reported by 197 (12.5\%) patients and severe adverse events were reported by $12(0.76 \%)$ patients. Overall, $76.3 \%$ of the patients and $78.4 \%$ of the investigators rated the test drug as effective.

Conclusions: The fentanyl matrix is believed to be effective for the treatment of pain, sleep disturbance and the impact upon daily and social activities, yet physicians should pay attention to the risks of abuse and the adverse events.

Key Words: Chronic pain, Spine, Transdermal fentanyl, Functional improvement

Received Jul 25, 2010; 1st Revised Aug 22, 2010; Accepted Aug 25, 2010

Corresponding author: Whan Eoh, MD, PhD

Department of Neurosurgery, Samsung Medical Center, Sungkyunkwan University School of Medicine,

50 Irwon-dong, Gangnam-gu, Seoul 135-710, Korea

Tel: +82-2-3410-3491, Fax:+82-2-3410-2447, E-mail: w.eoh@samsung.com 


\section{Introduction}

Non-opiates and non-steroidal anti-inflammatory drugs (NSAIDs) remain the standard medical treatments for nonmalignant low back pain due to their effectiveness for pain relief [1]. Acetaminophen therapy, although effective for the treatment of mild or moderate pain, is limited by the risk of hepatotoxicity when administered over extended periods or at doses over $4 \mathrm{~g} / \mathrm{day}$, and there is an increased bleeding tendency in patients taking warfarin combined with acetaminophen. NSAIDs are frequently used, but they are associated with ceiling effects and serious gastrointestinal bleeding.

Patients with more severe non-malignant chronic low back pain may require opioids for effective pain management. Opioids are known to participate in the pathway between the internal analgesic system and the macula nucleito spinal cord $[2,3]$. There have been several empirical observations and controlled trials demonstrating that opioids can provide significant relief for patients with nonmalignant chronic pain, with the resultant restoration of daily activities and this is accompanied by a low risk of serious adverse effects [4-8]. Transdermal fentanyl (TDF) matrix, which provides systemic delivery of fentanyl at a constant rate for 72 hours [9], has been shown to be effective for controlling pain in patients with chronic back pain [8-10]. The potential advantages of TDF include the convenience, continuous drug delivery and improved compliance [11]. This open-label trial was undertaken to evaluate the utility and safety of TDF for the treatment of chronic spinal disorder-related pain under conditions that approximate everyday medical practice.

The primary objective of this study was to determine the

Table 1. Patient characteristics of total and full data analysis set (FAS) patients

\begin{tabular}{|c|c|c|c|c|}
\hline \multirow{2}{*}{ Characteristics } & \multicolumn{2}{|c|}{ Total $(\mathrm{n}=1,576)$} & \multicolumn{2}{|c|}{ FAS $(n=1,252)$} \\
\hline & Mean & Std & Mean & Std \\
\hline Age (yr) & 62.8 & 12.9 & 63.29 & 12.66 \\
\hline Height (cm) & 161 & 9.5 & 160.96 & 9.85 \\
\hline Weight (kg) & 60.3 & 10.6 & 60.38 & 10.77 \\
\hline \multicolumn{5}{|l|}{ Gender } \\
\hline Male & \multicolumn{2}{|c|}{$606(38.5)$} & \multicolumn{2}{|c|}{$505(40.34)$} \\
\hline Female & \multicolumn{2}{|c|}{$970(61.5)$} & \multicolumn{2}{|c|}{747 (59.66) } \\
\hline
\end{tabular}

Values are presented as number $(\%)$.

FAS: Full data analysis set, Std: Standard deviation. impact of TDF on improving pain and function in patients with spinal disorder-related pain. The secondary objectives included improvement on the Korean version of the Oswestry Disability Index (K-ODI), sleep, everyday living, and social activities.

\section{Materials and Methods}

This was an open-label prospective, non interventional, electronic web-based study conducted between May and December 2008 at 63 orthopedic and neurosurgery-based clinics in Korea. The study was designed to determine the utility and side effects of the TDF patch (Durogesic ${ }^{\circledR}$ DTRANS, Janssen Korea, Seoul, Korea) for patients with spinal disorder-related chronic pain that was not adequately controlled by conventional conservative treatment.

The Institutional Review Boards at each hospital approved the study, and the study was carried out in accordance with the Declaration of Helsinki and the principles of good clinical practice. All the eligible patients provided written informed consent before entering the study.

\section{Patient selection}

The patients who were aged at least 20 years with spinal disorder-related chronic pain of more than 3 months duration, which remained severe (numeric rating scale $[\mathrm{NRS}]=$ 7) despite medication, were included in this study. Patients were excluded from the study if they had received fentanyl

Table 2. Diagnosis of total and FAS patients

\begin{tabular}{lcc}
\hline \hline Diagnosis & Total & FAS \\
\hline Degenerative spine disease & $970(61.6)$ & $765(61.6)$ \\
$\quad$ Spinal stenosis & $497(31.5)$ & $388(31.0)$ \\
$\quad$ Herniated intervertebral disc & $242(15.4)$ & $190(25.2)$ \\
Degenerative disc disease & $126(8.0)$ & $105(8.4)$ \\
$\quad$ Spondylolisthesis & $105(6.7)$ & $82(6.5)$ \\
Lumbar degenerative kyphosis & $69(4.4)$ & $57(4.6)$ \\
Fracture & $210(13.3)$ & $174(13.9)$ \\
$\quad$ Traumatic fracture & $71(4.5)$ & $58(4.6)$ \\
$\quad$ Osteoporotic fracture & $139(8.8)$ & $116(9.3)$ \\
Complex regional pain syndrome & $22(1.4)$ & $17(1.4)$ \\
Infection & $18(1.1)$ & $13(1.0)$ \\
Failed back surgery syndrome & $128(8.1)$ & $109(8.7)$ \\
Other deformity & $22(1.4)$ & $22(1.8)$ \\
Others & $137(8.7)$ & $95(7.6)$ \\
Total & $1,576(100.0)$ & $1,252(100.0)$ \\
\hline
\end{tabular}

Values are presented as number (\%).

FAS: Full data analysis set. 
matrix treatment within one month, they had a history of drug abuse, they were unable to use a transdermal system due to skin disease, they had a history of $\mathrm{CO}_{2}$ retention, hypersensitivity to opioid analgesics or they had a serious mental disease. Patients could also be excluded at the investigator's discretion.

\section{Medication}

TDF was administered every 72 hours, and it was generally started at a dose of $12 \mu \mathrm{g} / \mathrm{hr}$. The dose of TDF was adjusted by the investigator depending upon the level of pain relief. Opioids other than TDF could not be taken during the study, but as-needed analgesics (acetaminophen, NSAIDs), anxiolytics, antidepressants, anti-arrhythmic drugs, corticosteroids, anticonvulsants and preventative anti-emetics were permitted.

\section{Assessments}

In addition to the scheduled visits, all the patients were seen on demand. If emergency room visits or hospital admission were required, then the patient was removed from the study and he/she was treated routinely.

The patients were evaluated at baseline (day 1, Visit 1), on day 29 ( \pm 7 , Visit 2$)$ and day 57 ( \pm 7 , Visit 3). The primary efficacy variable was pain control, which was evaluated on an 11-point numeric rating scale $(0=$ no pain, $10=$ worst possible pain) during the 3 days preceding the visit. Post-treatment pain intensity scales were used to determine the percentage of the pain intensity difference (\% PID).

$\% \mathrm{PID}=$ [NRS (at baseline) - NRS (at final evaluation) $]$ $\times 100(\%) / \mathrm{NRS}$ (at baseline)

The secondary efficacy assessments included the K-ODI for assessing 10 domain scales: sleep disturbance (11-point numeric rating scale; $0=$ no disturbance, $10=$ worst possible disturbance) during the preceding 3 days; daily living and social activities (5-point verbal rating scale; $1=$ very good/no impairment, 5 = very poor/severe) during the preceding 3 days; the investigator and patient's global assessment (5-point rating scale; 1 = very poor, 5 = very good); the patient's preference and the clinical global impression (improvement: very much improved to very much worse).

Safety was evaluated by monitoring the patient's clinical condition and the spontaneously reported adverse events (the occurrence, nature, intensity and relationship to the study drug).

\section{Statistical analysis}

All the patients who received study medication and their data was recorded at baseline and at least once thereafter were included in the intention-to-treat population. All the pain severity and interference analyses were carried out using the full analysis set (The full analysis set is the group excluding the patients who violated major inclusion/exclusion criteria, who were not administered the study drug or whose efficacy data did not exist after the study drug administration).

The sample size was calculated using the hypothesis that $58 \%$ of the patients would record a decrease of more than $50 \%$ in pain intensity [12] after treatment with TDF.

The drug efficacy was evaluated in 1,252 patients (full data analysis set [FAS]), which included 1,037 patients who completed the last assessment in accordance with the study plan (the per-protocol population) and 215 patients who were excluded after the second assessment. Safety was assessed in the total population.

The data were analyzed using SAS ver. 9.1 (SAS Institute Inc., Cary, NC, USA). The Friedman test was used to analyze the change from baseline to the endpoint and the influence of the baseline values. The Wilcoxon signed rank test was used to compare the intra-group results and the results at each time point or at the endpoint with the baseline, when applicable. The correlation between the major efficacy evaluation parameters and changes in the K-ODI was evaluated using the Spearman Rho analysis. Statistical tests were interpreted at the 5\% significance level. Significant differences between baseline, week 4 and week 8 were assessed using the paired $t$-test.

\section{Results}

\section{Patients}

A total of 1,576 patients ( 970 men and 606 women) with an average age (standard deviation) of $62.8( \pm 12.9)$ years were enrolled and they received the study treatment, and 1,037 of these patients completed the study and were included in the per protocol population (Fig. 1). The reasons for discontinuation among the 539 patients (34.2\%) who withdrew from the trial during the treatment phase were lost to follow-up (225), adverse events (106), patient's decision (109), pain improvement (58) and other reasons (41).

The baseline demographics and diagnoses are presented 
in Tables 1 and 2, respectively. The most frequent diagnosis was degenerative spine disease including spinal stenosis. Eighty five point three percent of the patients had received previous treatment $(93.9 \%$ medication, $17.3 \%$ surgery, $11.2 \%$ physical treatment, $9.3 \%$ other treatments).

\section{Study medication}

The mean starting dose of TDF was $13.1 \mu \mathrm{g} / \mathrm{hr}( \pm 3.7$, $\mathrm{n}$ $=1,252)$. The average mean daily doses at weeks 4 and 8

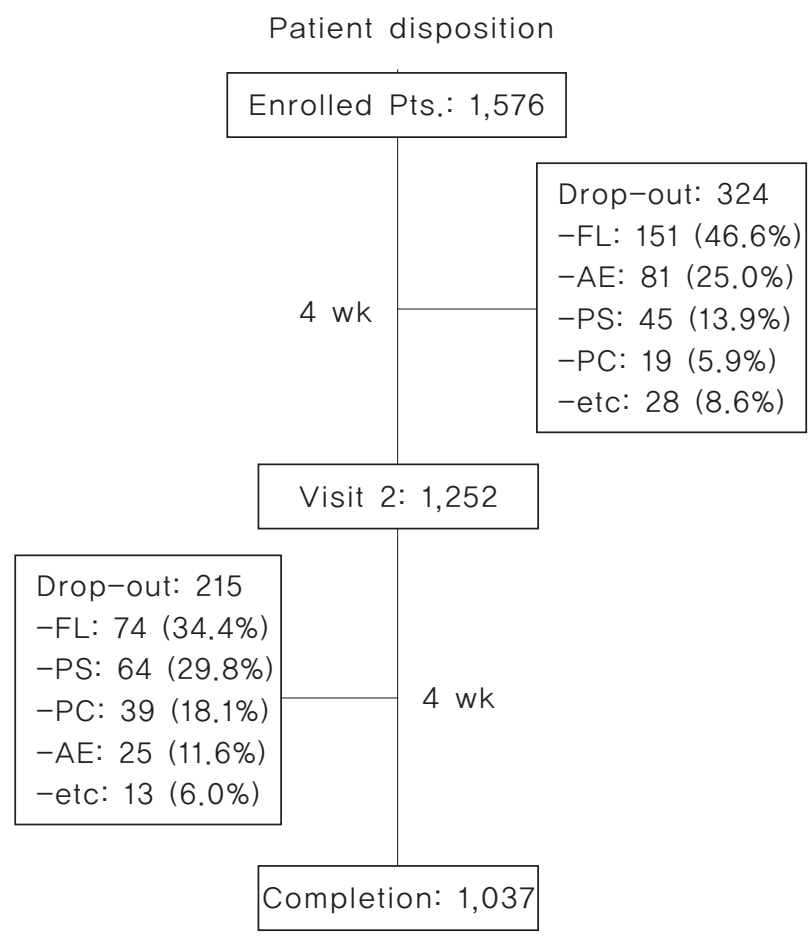

Fig. 1. Disposition of the study subjects. Pts: Patients, FL: Follow-up loss, AE: Adverse event, PS: Patient's selection, PC: Pain controlled. were $15.5 \mu \mathrm{g} / \mathrm{hr}( \pm 6.4, \mathrm{n}=1,252)$ and $16.0 \mu \mathrm{g} / \mathrm{hr}( \pm 7.3$, $\mathrm{n}=1,037$ ), respectively.

\section{Concomitant medications}

The concomitant medications used before and during the study are listed in Table 3. The most commonly used analgesics were Ultracet ${ }^{\text {TII }}$ (Janssen Korea) (33.5\%), gabapentin (13.3\%), and aceclofenac (8.8\%). A total of 1,405 patients received analgesic treatment before the study, 1,187 patients received continuous analgesic medication and 1,367 patients received concomitant medication during the study. There was no statistical difference between the doses of combination analgesics taken during the study. A total of 1,252 nonanalgesic preparations were prescribed during the study, including anti-emetics (domperidone, 160; metoclopramide, 7), laxatives (magnesium hydroxide, 6; magnesium oxide, 3 ) and anti-dizziness agents (dimenhydrinate, 2).

\section{(1) Pain intensity}

The pain intensity significantly decreased from 8.1 points at Visit 1 (Table 4) by $2.7 \pm 1.92$ points (33.5\%) to 5.4 at Visit $2(p<0.0001)$ and by $3.7 \pm 2.18$ points $(45.3 \%)$ at Visit 3 ( $p<0.0001$, Friedman test) (Fig. 2). The pain intensity decreased by an average of $45.3 \%$ among all the patients; for the patients recording a reduction of over $50 \%$, the average reduction was $66.1 \%$ (95\% confidence interval [CI], 65.2 to 67.1$)$. The pain intensity among the 1,252 patients in the FAS group showed an average reduction of $44.9 \%$; the patients with a reduction over $50 \%$ recorded an average reduction of $66.0 \%$ (95\% CI, 65.1 to 67.1$)$. Overall, $70.9 \%$ (95\% CI, 68.4 to 73.4 ) and $49.8 \%$ (95\% CI, 47.1 to 52.6) of the patients recorded reductions in pain intensity greater than $30 \%$ and $50 \%$, respectively.

Table 3. Previous and concomitant medication (analgesics) $(n=1,252)$

\begin{tabular}{|c|c|c|c|c|c|c|}
\hline \multirow[b]{2}{*}{ Analgesic types } & \multicolumn{2}{|c|}{ Previous medication } & \multicolumn{3}{|c|}{ Concomitant medication } & \multirow[b]{2}{*}{$\begin{array}{l}\text { Rate } \\
(\%)\end{array}$} \\
\hline & $\begin{array}{c}\text { No. of patients with } \\
\text { drug administered }\end{array}$ & $\begin{array}{l}\text { Rate } \\
(\%)\end{array}$ & $\begin{array}{c}\text { No. of patients with continuous } \\
\text { administration }\end{array}$ & $\begin{array}{l}\text { Rate } \\
(\%)\end{array}$ & $\begin{array}{c}\text { No. of patients with drawn from } \\
\text { administration }\end{array}$ & \\
\hline Non-opioid & 1,154 & 92.2 & $955(143)$ & 76.3 & 199 & 15.9 \\
\hline Weak-opioid & 45 & 3.6 & $34(5)$ & 2.7 & 11 & 0.9 \\
\hline Strong-opioid & 11 & 0.9 & $7(3)$ & 0.6 & 4 & 0.3 \\
\hline PRN analgesic & 9 & 0.7 & $7(1)$ & 0.6 & 2 & 0.2 \\
\hline Adjuvant analgesic & 186 & 14.9 & $173(41)$ & 13.8 & 13 & 0.1 \\
\hline Total & 1,405 & 112 & $1,176(191)$ & 93.9 & 229 & 18.3 \\
\hline
\end{tabular}

Analgesic newly administered during the study period is presented in parentheses.

PRN: Pro re nata. 


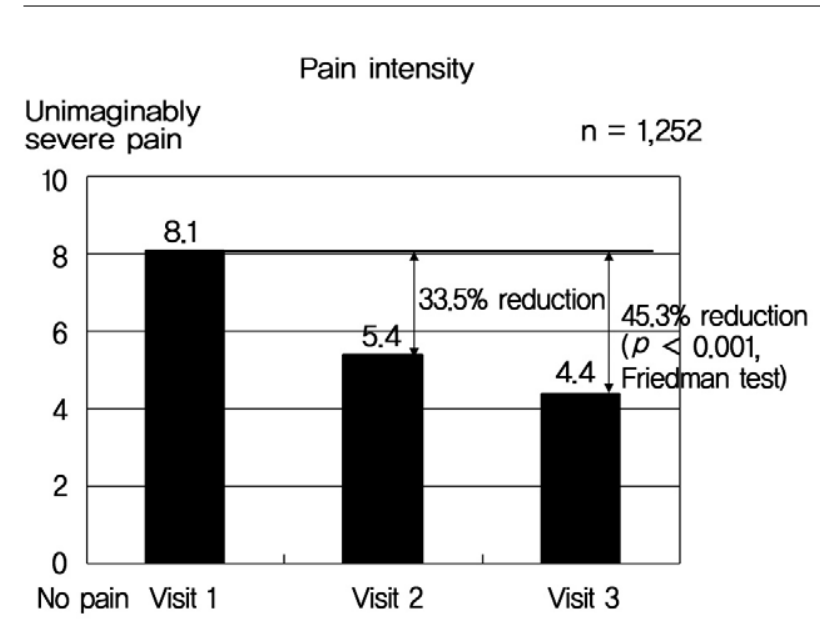

Fig. 2. Pain intensity during $8 \mathrm{wk}$ of treatment with transdermal fentanyl (full data analysis set).

Table 4. Baseline evaluations $(n=1,252)$

\begin{tabular}{lcc}
\hline \hline Parameter & Mean [max, min] & STD \\
\hline Pain intensity (NRS 0-10) & $8.1[7,10]$ & 1 \\
$\begin{array}{l}\text { Sleep disturbance due to } \\
\text { pain (NRS 0-10) }\end{array}$ & $6.5[0,10]$ & 2.7 \\
Pain interference on activity & $4.0[1,5]$ & 0.8 \\
$\quad \begin{array}{l}\text { of daily living (5 point scale: 1-5) } \\
\text { Pain interference on activity of }\end{array}$ & $4.0[1,5]$ & 0.9 \\
$\quad$ social life (5 point scale: 1-5) & & \\
Korean version of ODI score & 61.9 & 18.2 \\
\hline
\end{tabular}

STD: Standard deviation, NRS: Numerical rating scale, ODI: Oswestry Disability Index.

\section{(2) Sleep disturbance}

Sleep disturbance significantly decreased from 6.5 points at Visit 1 (Table 4) to 4.0 points at Visit 2 and to 3.1 points at Visit 3 ( $p<0.001$, Friedman test) (Fig. 3).

\section{(3) Disturbance in daily living activities}

Disturbance in daily living activities significantly decreased from 4.0 points at Visit 1 (Table 4) to 2.9 points at Visit 2 and to 2.5 points at Visit 3 ( $p<0.001$, Friedman test) (Fig. 4).

\section{(4) Disturbance in social activities}

Disturbance in social activities significantly decreased from 4.0 points at Visit 1 (Table 4) to 2.9 points at Visit 2 and to 2.5 points at Visit 3 ( $p<0.001$, Friedman test) (Fig. 5).
(5) $\mathrm{K}-\mathrm{ODI}$ and Korean version of the Neck Disability Index (K-NDI)

For the 1,216 patients who were analyzed, the K-ODI sig-

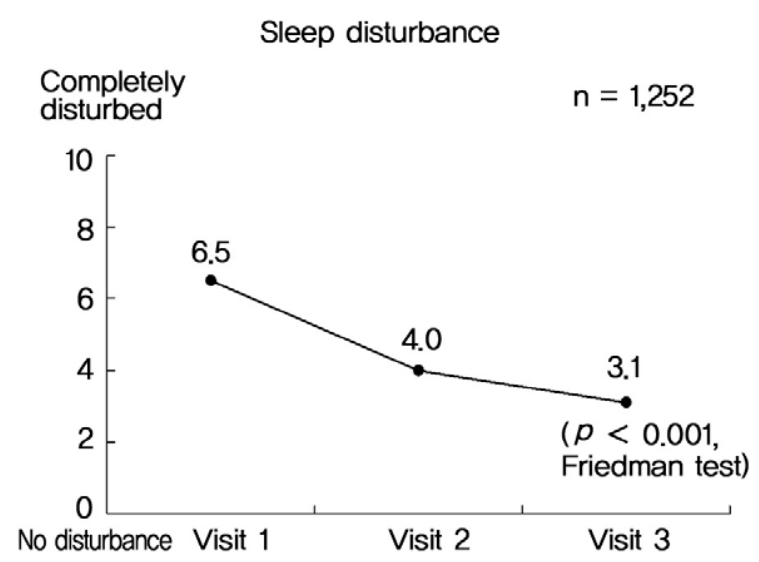

Fig. 3. Sleep disturbance during $8 \mathrm{wk}$ of treatment with transdermal fentanyl (full data analysis set).

\section{Activity of daily living}

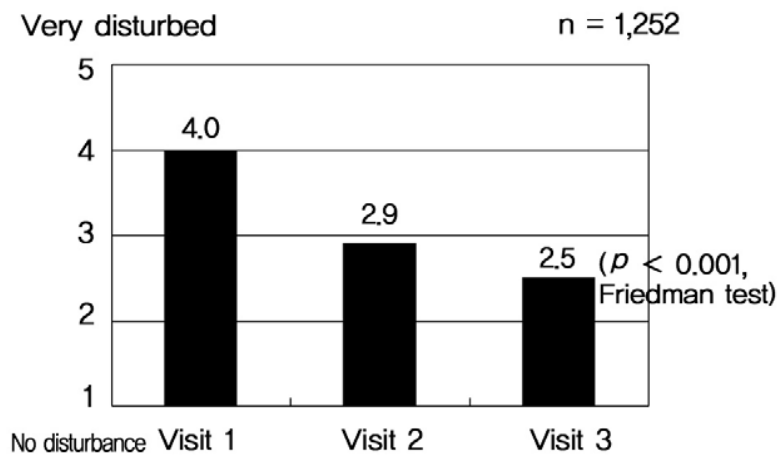

Fig. 4. The effect of 8 wk treatment with transdermal fentanyl upon daily living activities (full data analysis set).

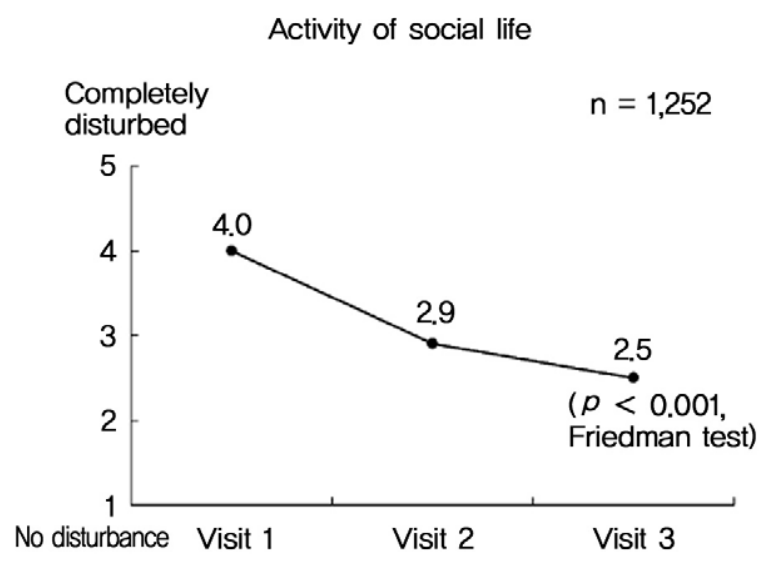

Fig. 5. The effect of 8 wk treatment with transdermal fentanyl upon social activities (full data analysis set).

nificantly decreased from $61.9 \%$ at Visit 1 to $45.8 \%$ at Visit 2 and to $38.2 \%$ at Visit 3 ( $p<0.001$, Friedman test) (Table 5).

Correlation analysis between the major efficacy evaluation parameters and the changes in the K-ODI demonstrated 
96 / ASJ: Vol. 5, No. 2, 2011

Table 5. Overall evaluation of K-ODI $(n=1,216)$

\begin{tabular}{|c|c|c|c|c|c|c|c|}
\hline \multirow{2}{*}{ Section } & \multicolumn{2}{|c|}{ Primary evaluation } & \multicolumn{2}{|c|}{ Secondary evaluation } & \multicolumn{2}{|c|}{ Tertiary evaluation } & \multirow{2}{*}{$\begin{array}{c}\text { Friedman test } \\
p \text {-value }\end{array}$} \\
\hline & No. & Ave (STD) & No. & Ave (STD) & No. & Ave (STD) & \\
\hline Pain intensity & 1,216 & $3.79(0.77)$ & 1,203 & $2.67(0.95)$ & 1,197 & $2.20(1.03)$ & $<0.001$ \\
\hline Personal care & 1,216 & $2.80(1.24)$ & 1,203 & $1.94(1.10)$ & 1,197 & $1.57(1.10)$ & $<0.001$ \\
\hline Lifting & 1,214 & $3.64(1.08)$ & 1,203 & $2.96(1.16)$ & 1,196 & $2.55(1.27)$ & $<0.001$ \\
\hline Walking & 1,214 & $2.68(1.34)$ & 1,205 & $2.02(1.21)$ & 1,195 & $1.70(1.21)$ & $<0.001$ \\
\hline Sitting & 1,215 & $2.82(1.24)$ & 1,206 & $2.06(1.07)$ & 1,194 & $1.68(1.06)$ & $<0.001$ \\
\hline Standing & 1,214 & $3.17(1.19)$ & 1,201 & $2.36(1.16)$ & 1,195 & $1.98(1.20)$ & $<0.001$ \\
\hline Sleeping & 1,215 & $2.67(1.40)$ & 1,204 & $1.75(1.16)$ & 1,197 & $1.38(1.10)$ & $<0.001$ \\
\hline Sexual life & 479 & $3.22(1.50)$ & 417 & $2.62(1.53)$ & 403 & $2.21(1.56)$ & $<0.001$ \\
\hline Social life & 1,212 & $3.07(1.19)$ & 1,202 & $2.38(1.16)$ & 1,195 & $2.02(1.21)$ & $<0.001$ \\
\hline Traveling & 1,212 & 3.15 (1.32) & 1,201 & $2.36(1.23)$ & 1,193 & $1.98(1.25)$ & $<0.001$ \\
\hline
\end{tabular}

K-ODI: Korean version of Oswestry Disability Index, Ave (STD): Average (standard deviation).

Table 6. Summary of main adverse events $(n=1,576)$

\begin{tabular}{lcccrrr}
\hline \multirow{2}{*}{ Adverse events } & \multirow{2}{*}{ No. $(\%)$} & \multicolumn{2}{c}{ Causality } & \multicolumn{2}{c}{ Severity } \\
\cline { 3 - 6 } & & $+(\%)$ & $-(\%)$ & Mild (\%) & Moderate $(\%)$ & Severe $(\%)$ \\
\hline Dizziness & $59(3.7)$ & $58(98.3)$ & $1(1.7)$ & $24(40.7)$ & $29(49.2)$ & $6(10.1)$ \\
Nausea & $58(3.7)$ & $58(100.0)$ & $0(0.0)$ & $26(44.8)$ & $28(48.3)$ & $4(6.9)$ \\
Vomiting & $54(3.4)$ & $54(100.0)$ & $0(0.0)$ & $16(29.6)$ & $27(50.0)$ & $12(20.4)$ \\
Itching & $16(1.0)$ & $16(100.0)$ & $0(0.0)$ & $7(43.8)$ & $7(43.8)$ & $2(12.4)$ \\
Headache & $9(0.6)$ & $9(100.0)$ & $0(0.0)$ & $3(33.3)$ & $5(55.6)$ & $1(11.1)$ \\
\hline
\end{tabular}

a linear relationship for all the parameters, including the rate of change of pain intensity $(r=0.66)$, the rate of change of sleep disturbance $(r=0.56)$, the rate of change of disturbances in daily living activities $(r=0.66)$, and the rate of change of disturbances in social activities $(r=0.63$; Spearman correlation test, all $p<0.001$ ).

The K-NDI, which was analyzed for 31 patients, significantly decreased from 58.3\% at Visit 1 to $46.3 \%$ at Visit 2 and to $38.6 \%$ at Visit $3(p<0.001$, Friedman test).

\section{(6) Safety analysis}

Of the 1,576 enrolled patients, 197 (12.5\%) patients reported adverse events and $12(0.76 \%)$ patients reported severe adverse events. Out of the 539 (25.8\%) patients who withdrew from the study, an adverse event was the major cause of drop-out for 139 patients. The most common symptoms included dizziness, nausea, vomiting, itching and headache; the incidence of vomiting was 3.4\% (20.4\% of the severe adverse events), but all other symptoms occurred in fewer than $4 \%$ of the patients (Table 6). Withdrawal syndrome was not reported and no deaths occurred during the study. There were no clinically significant changes of the vital signs during the study.
(7) Global assessments

According to the patients' overall assessment, $70.6 \%$ and $76.3 \%$ of patients rated the treatment as effective during Visits 2 and 3, respectively. The increase from Visits 2 to 3 was statistically significant ( $p<0.001$, McNemar test).

The investigator's overall assessment rated the treatment as effective in $73.9 \%$ and $78.4 \%$ of patients at Visits 2 and 3 , respectively. The increase from Visits 2 to 3 was statistically significant ( $p<0.001, \mathrm{McNemar}$ test).

The investigators' clinical global impression of 'improved/greatly improved' rose from 39.4\% during Visit 2 to $54.7 \%$ during Visit 3 . The increase in the response rate from Visit 2 to Visit 3 was statistically significant $(p<$ 0.001, McNemar test) (Fig. 6).

With respect to patient preference, $89.5 \%$ of the 1,146 respondents preferred TDF over their previously used analgesics. The reasons cited were constant pain relief (71.3\%), sleep improvement (11.9\%), greater convenience as a result of fewer drug administrations (12.7\%), reduction of opioid administration $(2.7 \%)$ and others (1.5\%). Notably, 876 $(84.5 \%)$ patients continued the use of the test drug even after the completion of the study. 
Clinical global impression - improvement

$$
\mathrm{n}=1,252
$$

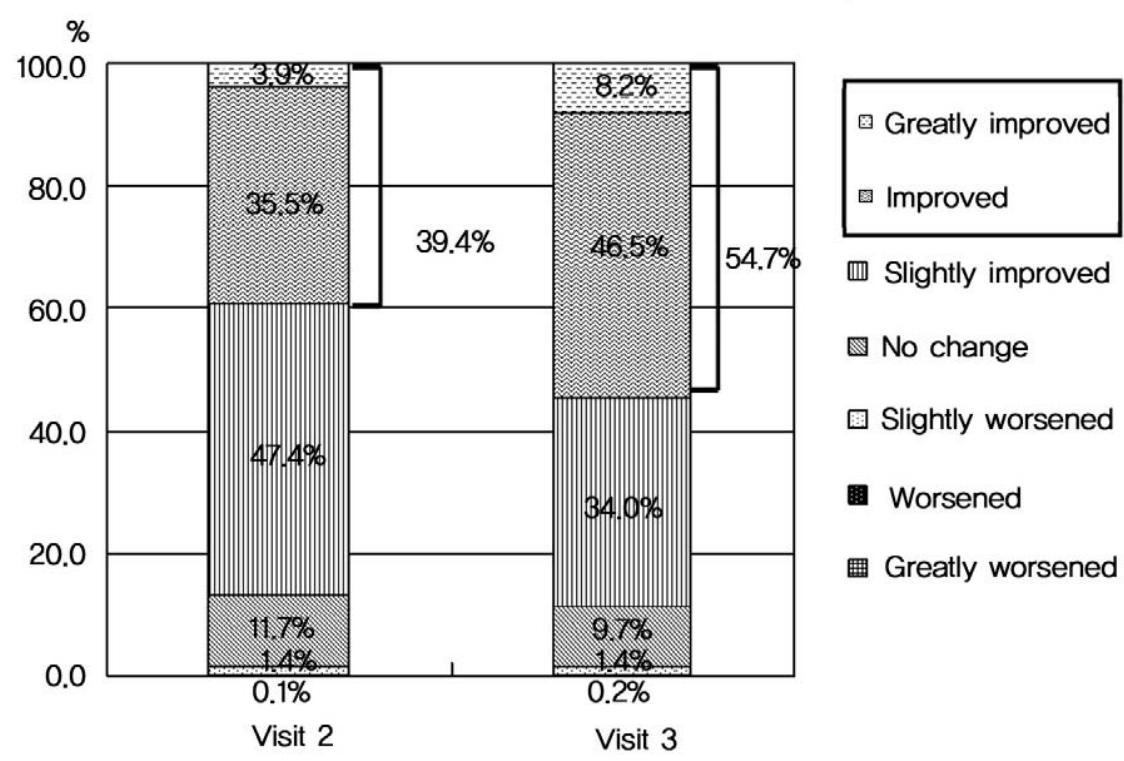

Fig. 6. Clinical global impression (per-protocol population).

\section{Discussion}

Although this study was only designed to evaluate the utility of TDF under routine conditions and to investigate different practical issues, it was valuable because it prospectively assessed the patients' reaction to TDF treatment in a large scale analysis and the patients were being treated currently for spine related chronic pain.

A statistically significant decrease in pain intensity was demonstrated during the study, and especially in terms of the degree of clinically significant pain reduction; the percentage of patients with over $30 \%$ and $50 \%$ reductions in pain intensity after treatment was $70.9 \%$ and $49.8 \%$, respectively. Furthermore, although this study was not placebocontrolled, over three quarters of the patients and investigators valued the drug to be 'greatly effective or effective;' more than half the patients reported that their pain was 'greatly improved or improved' compared to the pre-TDF treatment. The majority of patients preferred TDF over the previously used analgesics, and this was predominantly because of the stable pain relief during drug administration. Our results indicated that TDF provided significant pain relief in the majority of patients and that the degree of pain relief was significantly better compared to that of the previous medication. Yet there was not a significant difference in the frequency or dosage of concomitant medication between before and after TDF treatment; indeed, only 3 patients started using strong opioids. Needless to say, it would be preferable to exclude such concomitant medication when assessing the utility of TDF, but it is much more appropriate, from a pharmacological and ethical perspective, to allow the use of rescue medication for the treatment of break-through pain in patients who are taking long acting opioids such as fentanyl. The use of concomitant medication in this study did not decrease significantly because the dose of TDF administered at Visit 3 was only $16 \mu \mathrm{g} / \mathrm{hr}$, which is relatively low compared to the doses used in other studies. Nevertheless, the significant reduction in pain intensity irrespective of the low TDF dose in the patients with a NRS score of at least 7 and who had suffered from chronic pain for over 3 months despite previous treatment indicates the effectiveness of TDF.

In addition to the improvements in pain intensity, all the other parameters, including sleep disturbance due to pain and the degree of disturbance in daily living and social activities, significantly improved after week 8 as compared to pre-treatment. All 10 parameters measured with the KODI showed significant improvement compared with the pre-treatment values. The total K-ODI score was also significantly improved after treatment as compared with the score measured before the treatment started. This supports the notion that pain severely limits movement and that effective pain treatment could help patients to improve their 
daily living and social activities [13]. There was a positive correlation between the K-ODI scores and the rate of change of the pain intensity, the degree of sleep disturbance and the degree of disturbance in daily living and social activities following pain treatment, indicating that TDF improves not only pain treatment, but also the patient's function. This finding, which is one of the important goals for the treatment of patients with chronic pain, is therefore clinically significant.

Of the 1,576 patients who received at least one dose of the study drug, the percentage of patients who experienced adverse events was $12.5 \%$. In addition, the percentage of patients experiencing adverse events among the 1,037 patients who completed the 8 week study was only $5.6 \%$. This relatively low incidence and the nature of these adverse events recorded in this study were consistent with the other TDF trials and with strong opioids in general $[8,14]$. However, the relatively low frequency and intensity of adverse events may also have resulted from the low starting dose of TDF, which was chosen based on a previously reported observation that $12 \mu \mathrm{g} / \mathrm{hr}$ compared to $25 \mu \mathrm{g} / \mathrm{hr}$ does not lead to a big difference in pain relief, but the frequency of adverse events is much lower [15].

Tolerability is a very important issue with opioid treatment. Indeed, there have been cases where opioids with excellent analgesic properties failed to be used therapeutically due to the adverse events at the onset of treatment, and notably nausea, vomiting and dizziness. In this study, the withdrawal rate due to adverse events was highest during the early trial period and it was especially high at the initiation of the treatment. Concomitant treatment with domperidone (160 cases) and metoclopramide ( 7 cases) was prescribed for nausea and vomiting in this study. However, such adverse events tend to be self-limiting and transient and they can often be managed with prophylactic medications.

The incidence of severe adverse events at the initiation of therapy could hinder the use of a drug and so it is very important to use a low dose at the early stage, as was done in this study, in order to increase patient tolerability. Constipation occurred in only 9 out of 1,576 patients. These results are similar to those of the previous trials with TDF, and they support the benefits of TDF over oral treatments $[13,16]$. Indeed, TDF caused less constipation in patients with chronic non-cancer pain and cancer pain compared to that of sustained-release oral morphine [16,17]. Accordingly, the $12 \mu \mathrm{g} / \mathrm{hr}$ TDF patch used in this study was shown to be very effective [18] because it allows gradual dose titration based on the efficacy while lowering the incidence of adverse events [11].

The present study was an open-label trial of a relatively short duration, and it has several potential limitations, including the possibility of bias. Thus, the results should be interpreted with caution. Being an observational study, this study did not set the degree of pain control as the guideline for treatment. So, the treatment dosage of TDF was not increased systematically; this led to the NRS remaining at 4.1 despite 8 weeks of treatment and $57.4 \%$ of patients still complained of moderate to severe pain. Such findings could be the result of using a relatively low TDF dose of $16.8 \mu \mathrm{g} / \mathrm{hr}$ during the 8 week treatment period, and this may have led to a failure to administer the required dose of drug, as based on pain intensity. There were also limitations with respect to assessing the efficacy of TDF for the treatment of pain due to the allowed administration of concomitant analgesics. However, based on the observed improvements in the pain intensity through the introduction of TDF in the patients who had not benefited from previous analgesics for the treatment of chronic pain for 3 or more months, and there was no increase in the type or dose of TDF, it would not be unreasonable to conclude that TDF is effective for the treatment of pain.

\section{Conclusions}

In this study, the use of TDF led to additional pain control and improved functioning, including sleep and the daily and social activities. Thus, it can be concluded that TDF is effective for the treatment of spinal disorder-related chronic, severe pain. However, physicians should pay attention to cautiously prescribe TDF because opioids are associated with potentially serious harm, including opioid-related adverse effects and outcomes related to the abuse potential of opioids.

\section{Acknowledgements}

We would like to acknowledge the FEN-KOR-5019 Study Group and the other investigators for recruiting patients with chronic pain from a spine-related disorder.

This research was financially supported by a grant from Janssen Korea, Seoul, Republic of Korea, and Janssen's role was restricted to providing assistance to the investigators for the conception, conduct and analysis of this study. 
All the authors have received research funding from Janssen Korea.

\section{REFERENCES}

1. Cherkin DC, Wheeler KJ, Barlow W, Deyo RA. Medication use for low back pain in primary care. Spine (Phila Pa 1976) 1998;23:607-14.

2. Yang JY. The pathogenesis and medical treatment of spondylogenic pain. Asian Spine J 2010;4:57-63.

3. Woolf CJ. Recent advances in the pathophysiology of acute pain. Br J Anaesth 1989;63:139-46.

4. Portenoy RK. Opioid therapy for chronic nonmalignant pain: a review of the critical issues. J Pain Symptom Manage 1996;11:203-17.

5. Arkinstall W, Sandler A, Goughnour B, Babul N, Harsanyi Z, Darke AC. Efficacy of controlled-release codeine in chronic non-malignant pain: a randomized, placebo-controlled clinical trial. Pain 1995;62:169-78.

6. Moulin DE, Iezzi A, Amireh R, Sharpe WK, Boyd D, Merskey H. Randomised trial of oral morphine for chronic non-cancer pain. Lancet 1996;347:143-7.

7. Watson CP, Babul N. Efficacy of oxycodone in neuropathic pain: a randomized trial in postherpetic neuralgia. Neurology 1998;50:1837-41.

8. Simpson RK Jr, Edmondson EA, Constant CF, Collier C. Transdermal fentanyl as treatment for chronic low back pain. J Pain Symptom Manage 1997;14:218-24.

9. Jeal W, Benfield P. Transdermal fentanyl: a review of its pharmacological properties and therapeutic efficacy in pain control. Drugs 1997;53:109-38.

10. Ringe JD, Faber H, Bock O, et al. Transdermal fentanyl for the treatment of back pain caused by vertebral osteoporo- sis. Rheumatol Int 2002;22:199-203.

11. Langford R, McKenna F, Ratcliffe S, Vojtassák J, Richarz U. Transdermal fentanyl for improvement of pain and functioning in osteoarthritis: a randomized, placebo-controlled trial. Arthritis Rheum 2006;54:1829-37.

12. Dellemijn PL, Vanneste JA. Randomised double-blind active-placebo-controlled crossover trial of intravenous fentanyl in neuropathic pain. Lancet 1997;349:753-8.

13. Herrero-Beaumont G, Bjorneboe O, Richarz U. Transdermal fentanyl for the treatment of pain caused by rheumatoid arthritis. Rheumatol Int 2004;24:325-32.

14. Milligan K, Lanteri-Minet M, Borchert K, et al. Evaluation of long-term efficacy and safety of transdermal fentanyl in the treatment of chronic noncancer pain. J Pain 2001;2: 197-204.

15. Stumpf M, Bornhöovd K. Treatment-initiation with transdermal fentanyl $12.5 \mu \mathrm{g} / \mathrm{h}$ compared to transdermal fentanyl > $=25 \mu \mathrm{g} / \mathrm{h}$ (Durogesic ${ }^{\circledR}$ SMAT) in patients with chronic non-malignant pain. 5th Congress of the European Federation of IASP Chapters; 2006 Sep 13-16; Istanbul, Turkey. Amsterdam: Elsevier; 2006.

16. Donner B, Zenz M, Tryba M, Strumpf M. Direct conversion from oral morphine to transdermal fentanyl: a multicenter study in patients with cancer pain. Pain 1996;64: 527-34.

17. Clark AJ, Ahmedzai SH, Allan LG, et al. Efficacy and safety of transdermal fentanyl and sustained-release oral morphine in patients with cancer and chronic non-cancer pain. Curr Med Res Opin 2004;20:1419-28.

18. Choquette D, McCarthy TG, Rodrigues JF, et al. Transdermal fentanyl improves pain control and functionality in patients with osteoarthritis: an open-label Canadian trial. Clin Rheumatol 2008;27:587-95. 